\title{
Recent experiments at LUNA
}

\author{
Gianluca Imbriani ${ }^{* \dagger}$ \\ Physics Departement, University of Naples Federico II and INFN, Italy \\ E-mail: imbrianiena.infn.it
}

Cross section measurements for quiescent stellar H-burning are mainly hampered by extremely low counting rate and cosmic background. An effective method is to perform cross section measurements in an underground laboratory where cosmic ray flux is reduced by several orders of magnitude. LUNA experiments at the Gran Sasso National Laboratory in Italy represents the only experiments which are running in an underground environment. I will give a presentation of these experiments, mentioning the main results obtained thus far along with the status of the two experiments which are currently running at LUNA (Laboratory for Underground Nuclear Astrophysics): the measurements of the cross section of the reactions ${ }^{25} \mathrm{Mg}(\mathrm{p}, \gamma){ }^{26} \mathrm{Al}$ and ${ }^{15} \mathrm{~N}(\mathrm{p}, \gamma){ }^{16} \mathrm{O}$.

10th Symposium on Nuclei in the Cosmos

July 27 - August 12008

Mackinac Island, Michigan, USA

\footnotetext{
* Speaker.

${ }^{\dagger}$ For the LUNA collaboration.
} 


\section{Introduction}

The detailed description of most astrophysical and cosmological scenarios requires a very accurate knowledge of the many relevant nuclear processes. Information about nuclear mechanisms can be obtained in nuclear laboratories on sea level, although extremely low cross section measurements challenge the experimentalists. The aim of experimental nuclear astrophysics is to measure the nuclear reaction rates at the relevant astrophysical energies, which are very low. Indeed, in a stellar environment the energy available to nuclear species is very much lower than the Coulomb barrier [1]. Typical cross sections are of the order of $10^{-9}-10^{-12}$ barn or even lower, corresponding to experimental counting rate ranging from few events per day to few events per month, considering typical laboratory conditions. This means that the expected counting rate is prohibitively low and, even in those cases in which the event rate is compatible with human lifetime (i.e. in reactions between very light nuclei), the competition with cosmic background strongly hinders the acquisition of statistically significant results. There are three sources of background, i.e. cosmic rays, enviromental radioactivity and beam-target induced nuclear reactions. Each of these sources produces background of different nature and energy, so that each reaction to be studied deserves special care in suppressing the relevant background component. The suppression of the cosmic background can be accomplished by using large amounts of passive shielding, like that which can be found at the INFN underground laboratory at Gran Sasso (LNGS). Set up in the last decade, the LUNA (Laboratory for Underground Nuclear Astrophysics) collaboration continues to offer a globally unique facility for measuring low energy cross sections of astrophysical interest, installing two accelerators, respectively 50 [2] and $400 \mathrm{kV}$ [3], at LNGS.

\section{LUNA experiment}

During the first phase of the LUNA experiment using a home made $50 \mathrm{kV}$ accelerator, LUNA has measured the cross section of the nuclear processes ${ }^{3} \mathrm{He}\left({ }^{3} \mathrm{He}, 2 \mathrm{p}\right)^{4} \mathrm{He}[\llbracket]$ and $\mathrm{d}(\mathrm{p}, \gamma)^{3} \mathrm{He}$ [5], reaching for the first time the relevant astrophysical energy of the solar Gamow peak. The presence of a low energy resonance in ${ }^{3} \mathrm{He}\left({ }^{3} \mathrm{He}, 2 \mathrm{p}\right){ }^{4} \mathrm{He}$ was considered, before the SNO and Kamland results, as a possible nuclear explanation for the Solar Neutrino Problem. The LUNA experiment, measuring the cross section down to about $15 \mathrm{keV}$ [ $₫$ ] covered all the solar Gamow peak and excluded the existence of any resonance in the Gamow energy region. This important result showed that the solution of the Solar Neutrino Problem was not in the uncertainty of the Standard Solar Model. The reaction $\mathrm{d}(\mathrm{p}, \gamma)^{3} \mathrm{He}$ has been studied at LUNA also over the full energy range of the solar Gamow peak [5], i.e. as low as $2.5 \mathrm{keV}$. It represented the first case of a capture reaction studied underground, where the full advantage of an environment free of cosmic-ray induced background was appreciated. The results of high precision have a minor influence on the solar neutrinos, but are of critical importance for cosmology: during the era of big-bang nucleosynthesis the reaction was in competition with the universal expansion, which reduces the proton density. The reaction is thus important for the calculation of the primordial deuteron abundance [6].

With the scientific success of LUNA I the financial support improved significantly. Thus a commercial $400 \mathrm{kV}$ accelerator could be installed at LNGS (called LUNA II), which opened the possibility of improving our knowledge for other key reactions. The first reaction studied was 
the ${ }^{14} \mathrm{~N}(\mathrm{p}, \gamma){ }^{15} \mathrm{O}$, the slowest reaction in the H-burning $\mathrm{CNO}$ cycle, which determines the rate of this cycle. The reaction proceeds to the ground state as well as to several excited states of ${ }^{15} \mathrm{O}$ with different energy dependences. Thus, in one set of experiments Ge detectors in close and far geometry to a solid target were used to disentangle these dependences; the $\gamma$-ray detection efficiency was of course low (about $10^{-4}$ ). In the second experiment, a $4 \pi$ BGO crystal was used surrounding a windowless gas target with a nearly $70 \%$ detection efficiency. In the second experiment it was possible to extend the previous low-energy limit from $\mathrm{E}_{L}=260 \mathrm{keV}$ to $70 \mathrm{keV}$ [7, 8, 9, 10]. This energy is at the upper edge of the solar Gamow peak $\left(\mathrm{E}_{0}=30 \mathrm{keV}\right)$ but it is within the full Gamow peak e.g. for AGB (Asymptotic Giant Branch) stars. The combined data sets of LUNA II - together with the previous data obtained at higher energies - showed that the influence of a subthreshold resonance was overestimated in the earlier extrapolations for the capture into the ground state, while capture into excited states was properly extrapolated. Thus, the true rate is reduced by about a factor 2 for temperatures below $150 \cdot 10^{6} \mathrm{~K}$. The quoted rate reduction has several astrophysical implications on solar neutrino [11], age of globular clusters [8] and production of metal in Asymptopic Giant Branch [13].

The ${ }^{3} \mathrm{He}(\alpha, \gamma){ }^{7} \mathrm{Be}$ reaction was the second reaction measured by means of the LUNA II accelerator. It represents the largest uncertainty in the prediction of the flux of high-energy solar neutrinos. In previous experiments, its absolute cross section was derived from the observed flux of capture $\gamma$-rays as well as from the observed radioactivity of the residual nuclei ${ }^{7} \mathrm{Be}$, i.e. its electron capture to ${ }^{7} \mathrm{Li}$ with $\mathrm{T}_{1 / 2}=53$ days. Both results differ however by about $20 \%$ with a higher value from the ${ }^{7} \mathrm{Be}$ radioactivity, whose origin is not understood. Luna measured the cross section of this reaction for the first time by studying both the prompt $\gamma$-rays of approximately 1.6 and 1.2 $\mathrm{MeV}$ emitted directly by the capture reaction and the $500 \mathrm{keV} \beta$-delayed $\gamma$ from the ${ }^{7} \mathrm{Be}$ decay. Low energy data with extremely reduced systematic uncertainty were obtained ([14, 15, 16]) and the discrepancy between results obtained with the two experimental methods was resolved.

Presentely, we are finishing the data acquisition and analysis of two experiments: ${ }^{25} \mathrm{Mg}(\mathrm{p}, \gamma){ }^{26} \mathrm{Al}$ and ${ }^{15} \mathrm{~N}(\mathrm{p}, \gamma){ }^{16} \mathrm{O}$. The $\beta^{+}$decay of ${ }^{26} \mathrm{Al}_{\mathrm{gs}}{ }^{1}\left(T_{1 / 2}=7 \cdot 10^{5}\right.$ year) to the excited state of ${ }^{26} \mathrm{Mg}$ gives rise to a $1.8 \mathrm{MeV} \gamma$-ray, one of the most important lines for $\gamma$ astronomy. Indeed, the presence of ${ }^{26} \mathrm{Al}$ in the interstellar medium has been determined on the basis of direct observations of the $\gamma$-ray line at $1.809 \mathrm{MeV}$ from the COMPTEL [17, 18] and INTEGRAL [19, 20] satellites and from the observation of ${ }^{26} \mathrm{Mg}$ isotopic enrichment (extinct ${ }^{26} \mathrm{Al}$ ) in carbonaceous meteorites [21]. While the observations from COMPTEL and INTEGRAL provide evidence that ${ }^{26} \mathrm{Al}$ nucleosynthesis is still active on a large scale, the $\mathrm{Mg}$ isotopic variations show that ${ }^{26} \mathrm{Al}$ must have been produced not later than 4.6 billion years ago (time of the condensation of solar-system material). Any astrophysical scenario for ${ }^{26} \mathrm{Al}$ nucleosynthesis must be concordant with both observations. The ${ }^{26} \mathrm{Al}$ nuclides are produced mainly via the ${ }^{25} \mathrm{Mg}(\mathrm{p}, \gamma)^{26} \mathrm{Al}$ capture reaction, a member of the H-burning $\mathrm{MgAl}$ cycle, which operates in massive stars such as AGB's. The relevant temperature is $5 \times 10^{8} \mathrm{~K}$ corresponding to a Gamow energy of $100 \mathrm{keV}$. The presence of many states in the ${ }^{26} \mathrm{Al}$ isotope makes the study complicated; in the past the states down to $6496 \mathrm{keV}$, which correspond to a center of mass energy of $190 \mathrm{keV}$ in the ${ }^{25} \mathrm{Mg}+\mathrm{p}$ channel $(\mathrm{Q}=6306 \mathrm{keV})$, have been directly and indirectly

\footnotetext{
${ }^{1}$ About $80 \%$ of the released ${ }^{26} \mathrm{Al}$ is in the ground state, the remaining $20 \%$ goes into the $228 \mathrm{keV}$ isomeric state $\left(T_{1 / 2}=6 \mathrm{~s}\right)$.
} 
studied [22, 23, 24, 25, 26, 27, 28, 29, 30, 31]. The most used set up was a proton beam impinging into a ${ }^{25} \mathrm{Mg}$ target with a HPGe placed at $55^{\circ}$ degree, which gave the possibility to recognize all the cascade peaks. For the five lower energy states only upper limits have been indirectly deduced by the study of the ${ }^{25} \mathrm{Mg}\left({ }^{3} \mathrm{He}, \mathrm{d}\right){ }^{26} \mathrm{Al}$. The peculiarities of the $400 \mathrm{kV}$ LUNA facility [3] are particularly well suited for the study, where reaction $\gamma$-ray lines up to $\simeq 6.5 \mathrm{MeV}$ have to be measured with very low intensities. High beam intensities and high detection resolutions or efficiencies have to be coupled to high target stability and purity, which allow low beam-induced background; cosmic background is strongly suppressed by the mountain shielding and low intrinsic activity detectors are employed [32]. The aim of the experiment is to directly measure the branchings and the strenght of the resonances at $317 \mathrm{keV}$ and $190 \mathrm{keV}$ with an HPGe detector placed at $55^{\circ}$ and the strength of the resonances at 190,130 and $93 \mathrm{keV}$ using a summing crystal based on a $4 \pi$ BGO.

In stars that are slightly more massive than the sun, energy production is due mainly to the $\mathrm{CNO}$ cycle, which involves hydrogen fusing with various elements of carbon, nitrogen, and oxygen. The ratio between the reaction rate of the ${ }^{15} \mathrm{~N}(\mathrm{p}, \gamma){ }^{16} \mathrm{O}$ and the ${ }^{15} \mathrm{~N}(\mathrm{p}, \alpha){ }^{12} \mathrm{C}$ reactions directly determines the nucleosynthesis of the oxygen isotopes. An extensive measurement of the ${ }^{15} \mathrm{~N}(\mathrm{p}, \gamma){ }^{16} \mathrm{O}$ reaction was performed by Rolfs et al. [33]. In this study, it is showed that the reaction is dominated by the two $\mathrm{J}^{\pi}=1^{-}$resonances at $\mathrm{E}_{p}=338 \mathrm{keV}$ and $1028 \mathrm{keV}$, and also that the reaction is dominated by the cascade to the ground state. At energies corresponding to the interference region between the two resonances around $\mathrm{E}_{p}=500 \mathrm{keV}$ and for energies $\mathrm{E}_{p}<300$ $\mathrm{keV}$ the uncertainty of the data is rather large causing a large error in the extrapolated reaction rate. New precision experimental data at these energies are therefore extremely important. During 2007 LUNA has started a collaboration with the Nuclear Structure Laboratory of the Notre Dame University with the goal to investigate this reaction in a wide energy range. At the Notre Dame University the reaction has been measured in the energy range $\mathrm{E}_{p}=290-1900 \mathrm{keV}$, while at LUNA in the energy range of $\mathrm{E}_{p}=400-120 \mathrm{keV}$ with the same experimental setup. In this way the combination of the two measurements will cover a very large energy region and in particular the two most important interference regions, allowing a much better extrapolation to astrophysical energies. Furthermore the direct measurement at low energies, that will be performed at LUNA, will be an important test for the R-matrix extrapolation method. The Ti- ${ }^{15} \mathrm{~N}$ targets were fabricated in Karlsruhe using the reactive sputtering method. Using the very thin ${ }^{15} \mathrm{~N}(\mathrm{p}, \alpha \gamma){ }^{12} \mathrm{C}$ resonance at $430 \mathrm{keV}$, the targets were measured to be $8 \mathrm{keV}$ and $20 \mathrm{keV}$ thick. The data analysis is still in progress.

\section{References}

[1] C. Rolfs and W.S. Rodney, Cauldrons in the cosmos, University of Chicago Press (1988).

[2] U.Greife et al., Nucl.Instr.Meth. A 350(1994)327.

[3] Formicola, A., Imbriani, G., Junker, M., et al, Nucl.Instr.Meth. A 507(2003)609.

[4] R. Bonetti et al., Phys. Rev. Lett., 82(1999)5205.

[5] C. Casella et al, Nucl.Phys. A, 706(2002) 203-216.

[6] P.D. Serpico, et al., Journal of Cosmology and Astroparticle Physics, 12(2004)10.

[7] A. Formicola, G. Imbriani, H. Costantini et al, Phys.Lett.B, 591(2004)61-68. 
[8] G. Imbriani, H. Costantini, A. Formicola et al, Eur.Phys.Journal A, 25(2005)455-466.

[9] A. Lemut et al, Phys. Lett. B, 634(2006)483L.

[10] M.Marta, et al., Phys.Rev. C, 78(2008)022802.

[11] S. Degl'Innocenti et al., Phys. Lett. B 590(2004)13.

[12] G. Imbriani et al, $A \& A, 420(2004) 625$.

[13] F. Herwing, S. Austin and J.C. Lattanzio, Phys.Rev. C,73(2006)025802

[14] D. Bemmerer et al., Phys. Rev. Lett., 97 (2006) 122502

[15] Gy. Gyürky et al., Phys. Rev. C, 75 (2007) 35805

[16] F. Confortola et al., Phys. Rev. C, 75 (2007) 065803

[17] Diehl, R., Dupraz, C., Bennett, K., et al. A\&A, 298(1995)445.

[18] Knödlseder, J., Dixon, D., Bennett, K., et al. A\&A 345(1999)813.

[19] Winkler, C., Courvoisier, T.C., Di Cocco, G., et al. A\&A, 411(2003)L1.

[20] Diehl, R. et al. A\&A 411(2003)L451ÚL455.

[21] Wasserburg, G.J., in Protostars and planet II, edited by D.C. Black and M.S. Matthews (Universirty of Arizona Press, Tucson, 1985), p.703.

[22] A. E. Champagne, A. J. Howard and P. D. Parker. Nucl. Phys.A 402(1983)159.

[23] A. E. Champagne, A. J. Howard and P. D. Parker. Nucl. Phys.A 402(1983)179.

[24] P. M. Endt, P. de Wit and C. Alderliesten. Nucl. Phys.A 459(1986)61.

[25] A. E. Champagne, A. B. McDonald, T. F. Wang, A. J. Howard, P. V. Magnus and P. D. Parker. Nucl. Phys.A 451(1986)498.

[26] P. M. Endt and C. Rolfs. Nucl. Phys.A 467(1987)261.

[27] A. E. Champagne, A. J. Howard, M. S. Smith, P .V. Magnus and P. D. Parker. Nucl. Phys.A 505(1989)384.

[28] Ch. Iliadis, Th. Schange, C. Rolfs, U. Schröder, E. Somorjai, H. P. Trautvetter, P. M. Endt, S. W. Kikstra, A. E. Champagne, M. Arnould and G. Paulus. Nucl. Phys.A 512(1990)509.

[29] P. M. Endt. Nucl. Phys.A 521(1990)1.

[30] C. Iliadis, L. Buchmann, P. M. Endt, H. Herndl and M. Wiescher. Phys. Rev.C 53(1986)475.

[31] D.C.Powel, C.Iliadis, A.E.Champagne, S.E.Hale, V.Y. Hansper, R.A., R.A. Surman, K.D. Veal. Nucl. Phys.A 644(1998)263.

[32] D. Bemmerer et al, EpJA 24(2005)313.

[33] C. Rolfs, Nuclear Physics, A235(1974)450. 